\title{
Structure of yeast U6 snRNPs: Arrangement of Prp24p and the LSm complex as revealed by electron microscopy
}

\author{
RAMAZAN KARADUMAN, ${ }^{1}$ PRAKASH DUBE, ${ }^{2}$ HOLGER STARK, ${ }^{2,3}$ PATRIZIA FABRIZIO, ${ }^{1}$ \\ BERTHOLD KASTNER, ${ }^{1}$ and REINHARD LÜHRMANN ${ }^{1}$ \\ ${ }^{1}$ Department of Cellular Biochemistry, Max Planck Institute for Biophysical Chemistry, D-37077 Göttingen, Germany \\ ${ }^{2}$ Research Group 3D Electron Cryo-Microscopy, Max Planck Institute for Biophysical Chemistry, D-37077 Göttingen, Germany \\ ${ }^{3}$ Göttinger Zentrum für Molekulare Biologie, Universität Göttingen, D-37077 Göttingen, Germany
}

\begin{abstract}
Protein components of the U6 snRNP (Prp24p and LSm2-8) are thought to act cooperatively in facilitating the annealing of U6 and U4 snRNAs during U4/U6 di-snRNP formation. To learn more about the spatial arrangement of these proteins in S. cerevisiae U6 snRNPs, we investigated the structure of this particle by electron microscopy. U6 snRNPs, purified by affinity chromatography and gradient centrifugation, and then immediately adsorbed to the carbon film support, revealed an open form in which the Prp24 protein and the ring formed by the LSm proteins were visible as two separate morphological domains, while particles stabilized by chemical cross-linking in solution under mild conditions before binding to the carbon film exhibited a compact form, with the two domains in close proximity to one another. In the open form, individual LSm proteins were located by a novel approach employing C-terminal genetic tagging of the LSm proteins with yECitrine. These studies show the Prp24 protein at defined distances from each subunit of the LSm ring, which in turn suggests that the LSm ring is positioned in a consistent manner on the U6 RNA. Furthermore, in agreement with the EM observations, UV cross-linking revealed U6 RNA in contact with the LSm2 protein at the interface between Prp24p and the LSm ring. Further, LSmp-Prp24p interactions may be restricted to the closed form, which appears to represent the solution structure of the U6 snRNP particle.
\end{abstract}

Keywords: U6 snRNP; LSm tagging; electron microscopy; cross-linking

\section{INTRODUCTION}

The splicing of pre-mRNA to yield translatable mRNA involves the assembly of a multimegadalton complex, the spliceosome, on each intron to be excised. The spliceosome has a dynamic composition that includes five small nuclear RNAs (snRNAs), U1, U2, U4, U5, and U6, and more than 150 different proteins; many of these proteins are organized as small nuclear ribonucleoprotein (snRNP) complexes. The ordered assembly of the spliceosome is followed by the catalytic steps of splicing and then the dissociation of the spliceosome into its components, which are reused in subsequent splicing events (Burge et al. 1999; Jurica and Moore 2003; Will and Lührmann 2006).

Reprint requests to: Reinhard Lührmann, Department of Cellular Biochemistry, Max Planck Institute for Biophysical Chemistry, D-37077 Göttingen, Germany; e-mail: reinhard.luehrmann@mpi-bpc.mpg.de; fax: 49-551-201-1197.

Article published online ahead of print. Article and publication date are at http://www.rnajournal.org/cgi/doi/10.1261/rna.1369808.
The core of the spliceosome consists of a highly complex, dynamic, and protein-dependent network of RNA molecules, in which both snRNA-snRNA and snRNA-pre-mRNA interactions are present. The most highly conserved snRNA is the U6 snRNA, and it is believed to be the most important participant in the catalysis of the two transesterification steps that make up the splicing reaction. Furthermore, U6 snRNA is subject to unusually large conformational changes during the splicing cycle. It enters the spliceosome base paired through two double helices (stems I and II) with the U4 snRNA (Brow and Guthrie 1988; Wolff and Bindereif 1993; Fortner et al. 1994) in the so-called U4/U6-U5 tri-snRNP. However, before the actual splicing of the pre-mRNA takes place, the U4 snRNA dissociates and the U6 snRNA base pairs with the U2 snRNA and the pre-mRNA. After the catalytic steps, this binding is likewise disrupted, and the U6 snRNA dissociates as a free U6 snRNP. The U6 snRNA is finally recycled by undergoing base-paired association with the U4 snRNA, forming the U4/U6 di-snRNP, and this in turn associates with the U5 snRNP to form the tri-snRNP, which can 
participate in another cycle of splicing (Staley and Guthrie 1998; Brow 2002; Nilsen 2003).

The mechanism of the first step in the functional cycle of U6 snRNA-i.e., base-pairing with U4 snRNA-is at present only partly understood. It is known that a necessary condition for this step is the presence of the protein components of the U6 snRNP. These include the seven structurally related LSm proteins (in yeast, denoted LSm2$\mathrm{p}-\mathrm{LSm} 8 \mathrm{p}$ ) and a single additional protein designated in the yeast S. cerevisiae as Prp24p and in human as p110 or SART3 (Shannon and Guthrie 1991; Stevens et al. 2001; Bell et al. 2002). Prp24p has a molecular weight of $50 \mathrm{kDa}$ and consists mainly of four RNA recognition motif (RRM) domains, of which at least two contribute to its binding to the U6 snRNA (Shannon and Guthrie 1991; Vidaver et al. 1999; Rader and Guthrie 2002; Kwan and Brow 2005). The crystal structure of a large Prp24p fragment consisting of the three N-terminal RRMs was recently solved (Bae et al. 2007). The seven LSm proteins of the U6 snRNP all belong to the Sm class of proteins that consist mainly of a characteristic structural motif, the Sm domain (Hermann et al. 1995; Séraphin 1995). Both the seven LSm proteins and seven canonical Sm proteins form an annular complex 7-8 $\mathrm{nm}$ in diameter and $\sim 3 \mathrm{~nm}$ in thickness, as shown by both $\mathrm{X}$-ray crystallography and electron microscopy (Achsel et al. 1999; Kambach et al. 1999; Mura et al. 2001; Zaric et al. 2005). The sequence homology between the LSm and seven canonical Sm proteins (which are found in the snRNAs U1, U2, U4 and U5) (Séraphin 1995) and the results of two-hybrid interaction analyses and coexpression studies (Pannone et al. 2001; Zaric et al. 2005) indicate that the order of the LSm proteins in the ring is likely to be 2-3-6-5-7-4-8.

The structure of the U6 snRNA in the U6 snRNP is defined by a $5^{\prime}$ and a $3^{\prime}$ stem-loop. The latter has a stem extension (also called the telestem) that is separated from the terminal stem-loop by a large asymmetric internal loop (Fig. 1A; Brow and Vidaver 1995; Vidaver et al. 1999; Ryan et al. 2002; Karaduman et al. 2006). The $3^{\prime}$ end of the U6
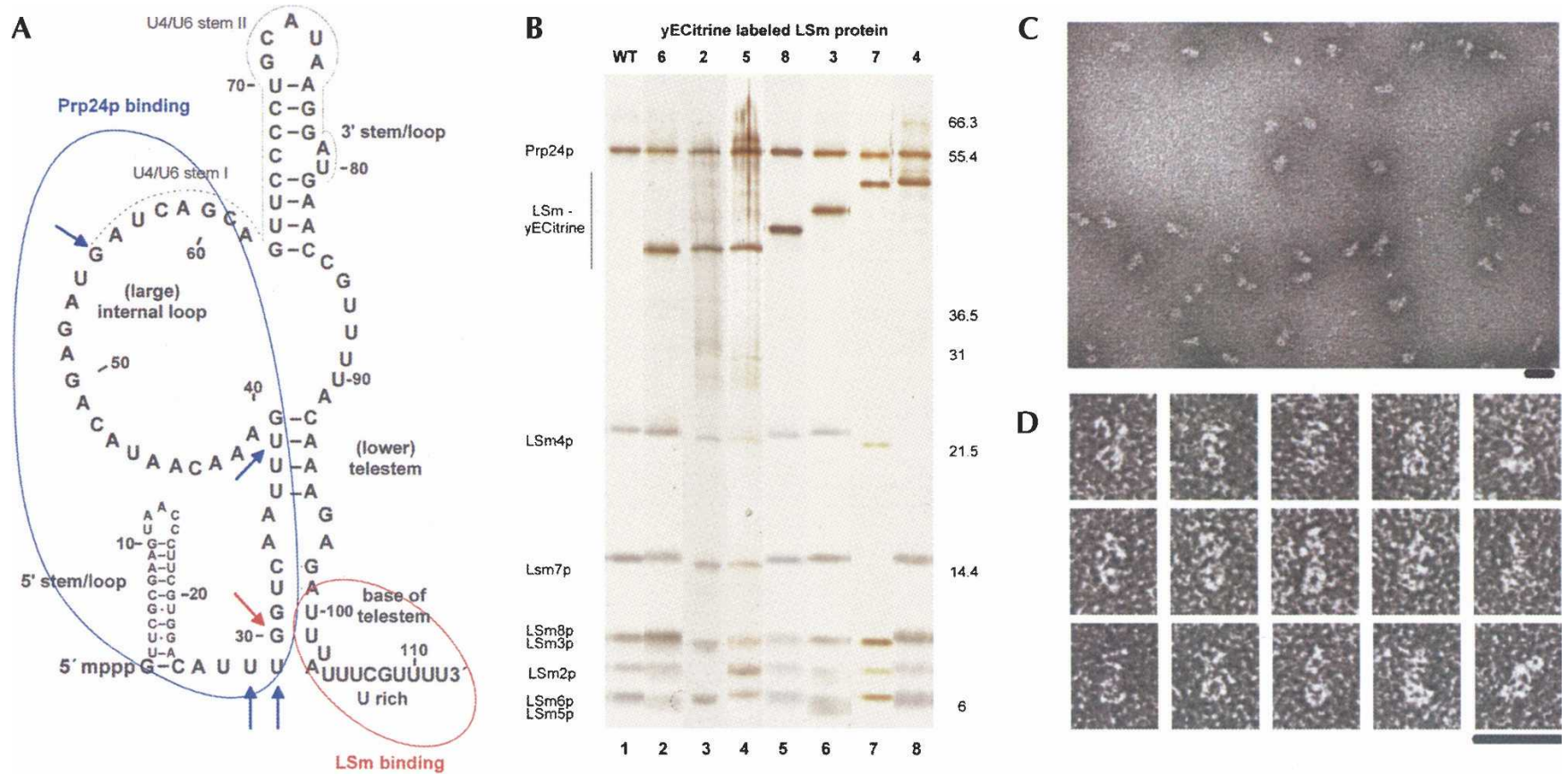

FIGURE 1. Protein composition and EM structure of the S. cerevisiae U6 snRNP. (A) Secondary structure of U6 snRNA in the U6 snRNP. The U6 snRNA is shown with the secondary structure as determined by Karaduman et al. (2006). Important structural features are labeled. Arrows indicate nucleotides that in previous work (Karaduman et al. 2006) were found to be UV-cross-linked to Prp24p (blue) or to an unidentified LSm protein (red). The blue and the red outlines indicate U6 snRNA regions that are covered and protected by Prp24p and the LSm proteins, respectively. U6 snRNA sequences involved in base pairing with U4 snRNA in the U4/U6 di-snRNP are indicated by dotted lines and are labeled U4/U6 stem I and stem II, respectively. (B) Protein composition of purified U6 snRNPs. Proteins from purified U6 snRNPs were separated by SDS-PAGE and stained with silver. (Lane 1) Proteins of the wild-type particle with a TAP-tagged version of Prp24p; (lanes 2-8) U6 snRNPs containing a single tagged LSm protein (as indicated above each lane). The positions of molecular weight markers are indicated on the right and protein identities on the left. The molecular weights of the tagged LSm proteins are increased by $30 \mathrm{kDa}$ due to the yECitrine; note the absence of untagged protein in each case (this is seen unambiguously for LSm2p, LSm7p, and LSm4p, but is less clear for the others, owing to pairwise overlap of the unmodified proteins). (Lanes 3,4) The protein composition of U6 snRNPs analyzed directly after TAP purification, while particles analyzed in all other lanes had additionally undergone glycerol-gradient centrifugation. $(C)$ A typical electron micrograph showing a field of U6 snRNPs. After purification on a glycerol gradient, U6 particles were directly adsorbed onto a carbon carrier film and negatively stained with uranyl formate. Scale bar, $20 \mathrm{~nm}$. (D) Gallery of selected U6 images. U6 particles were oriented with the round LSm domain at the bottom and the angular Prp24p domain at the top. In the images of the far right-hand column, the LSm ring is probably seen from the side. Scale bar, $20 \mathrm{~nm}$. 
RNA ends with a single-stranded pyrimidine-rich sequence that is essential for the binding of the LSm proteins (Achsel et al. 1999). The canonical RNA-binding site at the surface of the inner hole of the LSm ring is very probably the binding site for the pyrimidine-rich sequence at the $3^{\prime}$ end of U6 snRNA (Achsel et al. 1999; Vidal et al. 1999). How the pyrimidine-rich sequence is arranged in the presumed seven nucleotide-binding pocket, and whether this sequence has a fixed or preferred position on the LSm ring, remains unclear.

In yeast U6 snRNP, the principal RNA-binding site of Prp24p is the large internal loop, as has been shown by chemical "footprinting" and by UV cross-linking (Karaduman et al. 2006). In addition, almost all of the nucleotides on the $5^{\prime}$ side of this loop, including the $5^{\prime}$ stem-loop, are protected from hydroxyl-radical attack by bound Prp24p (Fig. 1A; Karaduman et al. 2006).

In accordance with the separation of their binding sites on U6 snRNA (Fig. 1A), Prp24p and the LSm complex bind independently to U6 (Ghetti et al. 1995; Ryan et al. 2002; Karaduman et al. 2006). However, the binding of Prp24p is strongly stimulated by the presence of the LSm proteins (Vidal et al. 1999; Ryan et al. 2002). A similar cooperative interaction was found in studies investigating the binding of the U4 snRNA to the U6 RNA. Here, the presence of Prp24p or the LSm complex stimulates U4/U6 binding; if both are present, the formation of the U4/U6 duplex is considerably more efficient (Rader and Guthrie 2002; Ryan et al. 2002; Verdone et al. 2004). Compared with the naked U6 snRNA, the U6 in the U6 snRNP has a conformation that is much more favorable for binding U4 snRNA (that is, more of the U4-binding nucleotides are single stranded) (Karaduman et al. 2006). Thus, Prp24p and the LSm ring may act as molecular chaperones.

This observed cooperative effect was attributed, on the basis of yeast two-hybrid interaction analyses, to direct interactions between Prp24p and the LSm proteins (FromontRacine et al. 2000; Rader and Guthrie 2002). It was found that these interactions depended upon a highly conserved sequence motif of 12 amino acids, located directly at the $\mathrm{C}$ terminus of the Prp24p protein (Rader and Guthrie 2002). This motif is also present in the human ortholog p110 (Bell et al. 2002). By far the strongest interactions with the wild-type Prp24p, and also the greatest decrease in interaction upon deletion of the C-terminal motif, were observed for the LSm proteins 5, 7, and 8 (Fromont-Racine et al. 2000; Rader and Guthrie 2002).

To obtain insight into the way in which the two distinct domains of the U6 snRNP (i.e., Prp24p bound to the U6stem-loop and the LSm 2-8 ring complexed to the U6 snRNA 3 ' end) are organized structurally in the U6 snRNP, we purified these particles from the yeast S. cerevisiae and examined them with and without prior stabilization by chemical cross-linking in solution in the electron microscope. Without fixation, an open form was predominately observed in which the LSm ring and the Prp24p protein were recognizable as separate morphological domains, while a compact form having these two domains in closer proximity to each other predominated after chemical crosslinking. To determine the positions of the LSm proteins with respect to the Prp24p domain, we developed an efficient genetic tagging method for locating the LSm proteins by electron microscopy (EM). The protein tag also allowed us to identify LSm2p as the LSm protein that can be cross-linked to the U6 snRNA outside of the latter's 3 '-terminal pyrimidine-rich region. The results presented here show that the U6 snRNA is bound in a defined manner to the LSm ring, with LSm2p at the site where the RNA links Prp24p to the LSm ring.

\section{RESULTS}

\section{Organization of U6 snRNP into two morphologically distinct domains}

For structure determination by EM, U6 snRNPs were isolated from extracts of a $S$. cerevisiae strain containing a TAP-tagged Prp24 protein by means of the tandem affinity purification (TAP) procedure (Puig et al. 2001; Karaduman et al. 2006) and then further purified by glycerol-gradient centrifugation. This yielded highly pure U6 snRNPs, which consisted of U6 snRNA, the Prp24p protein, and the seven LSm proteins LSm2p-LSm8p (Fig. 1B, lane 1). In a first experiment, particles in the U6-snRNP-containing gradient fractions were adsorbed to carbon films immediately after gradient fractionation, negatively stained, and examined under the electron microscope.

The U6 snRNP exhibits a somewhat elongated shape, 14-16 $\mathrm{nm}$ in length and 7-9 $\mathrm{nm}$ in diameter (Fig. 1C). Even at the low magnification of the general field view, a two-domain structure can be discerned in many particles. At higher magnification (Fig. 1D) one of these domains appears round (in the figures this domain is oriented at the bottom), while the other is more angular or "cornered" in shape. With a diameter of 7-8 $\mathrm{nm}$ and an often-visible central spot of stain, the round substructure possesses the typical appearance of the ring-shaped Sm or LSm complexes (Kastner et al. 1990; Achsel et al. 1999; Zaric et al. 2005). This substructure, therefore, is assigned to the LSm2p-LSm8p complex of U6 snRNP. Consequently, the upper domain is taken to be the other major protein component of U6 snRNP: the Prp24 protein. Subsequent tagging of the LSm proteins followed by EM (see below) further supported this assignment.

To analyze structural details of the U6 snRNPs, image processing was performed for each domain separately. A selection of typical class averages obtained in this way is shown in Figure 2A for the classification performed on the basis of the (upper) Prp24p domain and in Figure 2B for that performed with the (lower) LSm ring domain. 
A
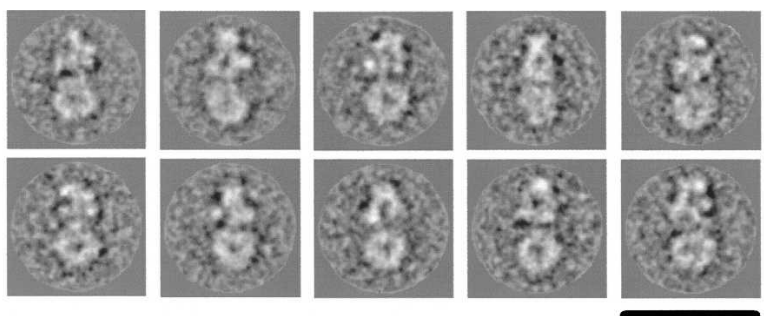

B
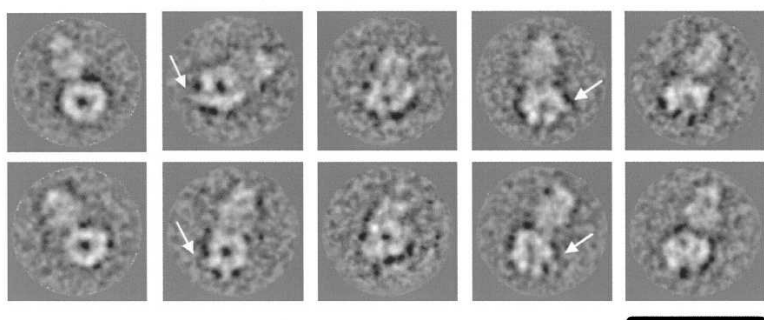

FIGURE 2. Structural details of the U6 snRNPs. To analyze structural details of the purified U6 snRNPs, image processing was performed for each domain separately. U6 snRNP images were classified according to the Prp24p domain structure after masking the LSm domain $(A)$ or according to the LSm ring structure after masking the Prp24p domain $(B)$. The class members were finally averaged without the mask, so that the whole particle image is incorporated in the averages shown. Small characteristic protrusions of the LSm ring $(B)$ are indicated by arrows. Scale bars, $20 \mathrm{~nm}$.

Processing of the whole-particle images improved the resolution much less, and then generally of only one of the two domains (data not shown). The most immediately plausible explanation for this is the presence of a flexible connection between the LSm ring and Prp24p in the U6 snRNP.

The structural organization of Prp24p and LSm into morphologically distinct domains reflects the two separate binding regions of Prp24p and the LSm protein on the U6 snRNA (Fig. 1A; Karaduman et al. 2006). The two domains may be in physical contact with each other, as the projections seen in the micrographs suggest that they are directly adjacent to one another.

\section{Topographical tagging of the LSm proteins with yECitrine}

The apparent contact between the Prp24p domain and the LSm ring suggests in principle that Prp24p might interact directly with one or more LSm proteins. There are several possibilities for such an interaction, depending upon whether the LSm ring adopts a consistent position relative to the Prp24p domain. We, therefore, investigated the organization of the LSm ring in the U6 snRNP. To do so, we developed a new procedure for locating proteins in EM images using the yeast system. The protein to be located is extended by an additional protein domain (a tag) at its $\mathrm{C}$ terminus. This is achieved genetically by introducing the tag sequence by homologous recombination into a yeast strain additionally expressing TAP-tagged Prp24p (Puig et al. 2001). This ensures that each copy of the protein to be located is expressed with the tag. Location experiments performed with antibodies, in contrast, usually yield only a subpopulation of labeled particles.

For the relatively small U6 snRNP particle, we chose as a tag the 30-kDa yECitrine protein (Sheff and Thorn 2004) as it is large enough $(\sim 4 \mathrm{~nm})$ to be recognized directly in EM images, but small enough to allow its position to be determined precisely, and also might have little or no effect on protein function. We prepared U6 snRNPs in which each LSm protein (in turn) was tagged with yECitrine. The differentially tagged U6 snRNPs were purified via Prp24pTAP. Analysis of their protein content showed that highly pure particles were isolated in each case and that the tagged protein's molecular weight increased by $30 \mathrm{kDa}$, corresponding to the mass of the yECitrine tag (Fig. 1B, lanes 2-8). In the LSm2, LSm3, LSm5, LSm6, and LSm7 proteins, the yECitrine sequence is located directly at the Sm domain, while in LSm8p and LSm4p, the tag and the Sm domain are somewhat farther apart, as these proteins contain, respectively, 30 and 100 amino acid residues between their Sm domain and $\mathrm{C}$ terminus.

The various tagged U6 snRNPs were examined under the electron microscope. With LSm7p-tagged U6 snRNPs, the single-particle images in the general field (Fig. 3A) clearly appear longer $(\sim 20 \mathrm{~nm})$ than the untagged particles (Fig. 1C). At higher magnification, many images (oriented as in Fig. 1) show the typical ring structure of the LSm complex (Fig. 3B), with two separate, differently shaped peripheral structures: a larger, angular structure very similar to the Prp24p domain and a smaller compact structure of about $4 \mathrm{~nm}$ diameter, which has the size and shape expected of the $y$ ECitrine protein (see the arrows in Fig. 3B). In the images, the two peripheral structures are almost invariably located on opposite sides of the LSm ring. Therefore, the yECitrine domain and, additionally, LSm7p, to which it is fused, are well separated from the Prp24p domain. In almost all images, the LSm7p tag is directly opposite the site where Prp24p contacts the LSm ring. This result suggests that the Prp24 protein has a consistent position on the LSm ring; if its position were highly variable, it would in some cases be found closer to the tag of the LSm7 protein. A highly similar result was obtained with LSm5p-tagged U6 snRNPs (Fig. 3C,D). Here again, the location of the LSm5p tag and that of Prp24p on the LSm ring are far apart, and more or less diametrically opposed. These results are consistent with the proposed order of LSm proteins in the LSm ring that is based on currently available biochemical data (see Introduction), in which LSm5p is a direct neighbor of LSm7p.

\section{The LSm ring is uniformly positioned with respect to Prp24p}

In U6 snRNPs containing tagged LSm6p and LSm8p, the proposed next-but-one neighbors of LSm7p, the yECitrine 


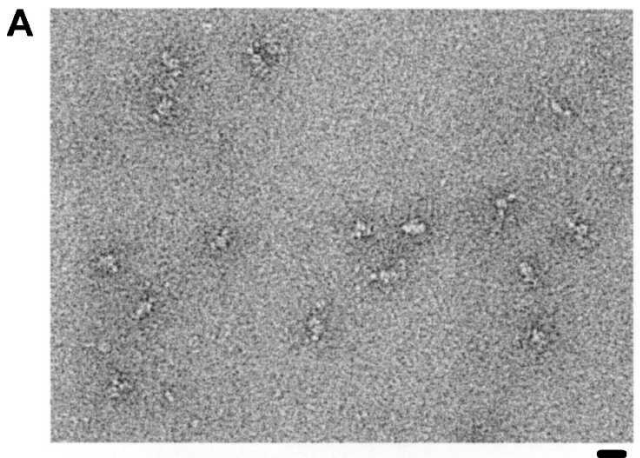

B

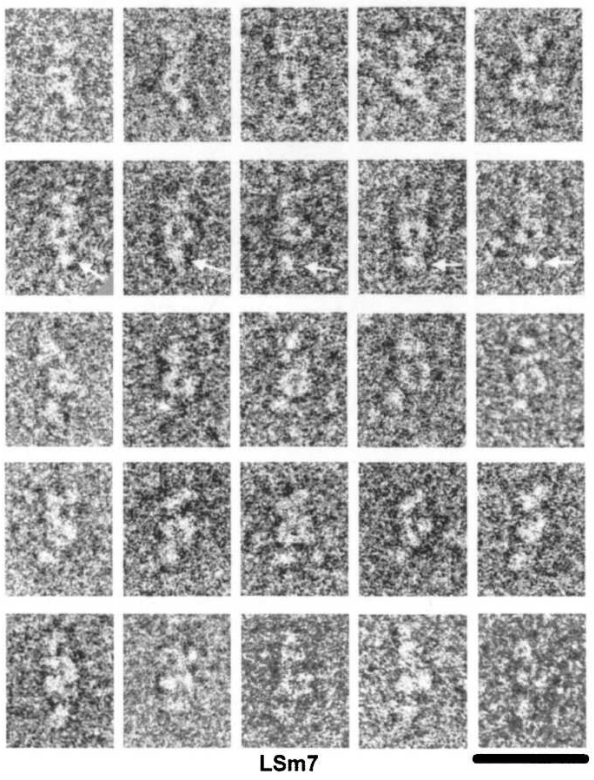

C

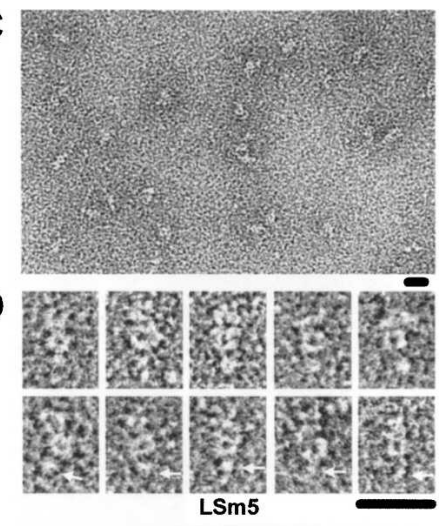

E

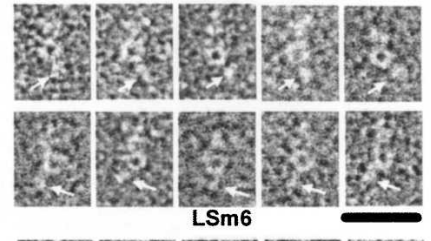

$\mathbf{F}$

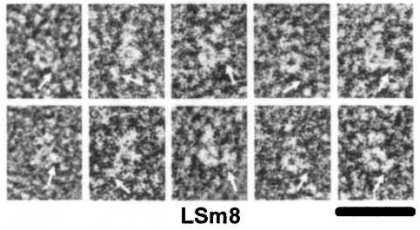

G

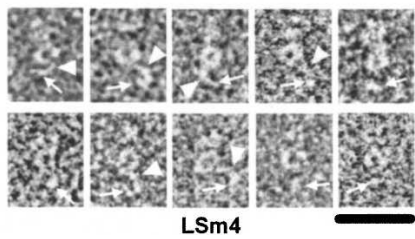

FIGURE 3. Electron microscopy of U6 snRNP with yECitrine-tagged LSm proteins. $(A, C)$ Fields from typical micrographs with proteins LSm7 $(A)$ or LSm5 $(C)$ tagged. $(B, D-G)$ Galleries of selected images of U6 snRNPs containing a tagged LSm7, LSm5, LSm6, LSm8, or LSm4 protein, as indicated below each gallery. In addition to the round LSm domain and the angular Prp24p domain (oriented upward in all galleries), a third small globular domain (the yECitrine tag) about $4 \mathrm{~nm}$ in diameter is visible (some are indicated by arrows). Note that some of the figures appear as mirror images of others, because the LSm ring can lie with either face in contact with the carbon film. For LSm4p $(G)$, the tag at the C terminus is often seen at some distance from the LSm ring. A region of stain low density, linking the tag to the LSm ring can be recognized in several images (labeled with arrowheads). The distance between the yECitrine tag and the Prp24p domain was estimated visually for particles with a clearly visible LSm ring. By classifying these into rough distance categories we observed characteristic (relative) distances for each tag position. Scale bars, $20 \mathrm{~nm}$.

Consistent with this, in EM images of U6 snRNPs containing tagged LSm4p, the yECitrine tag often appeared to be located at a distance from the periphery of the LSm ring, and it was sometimes difficult to distinguish it from the background noise. However, in some images a physical connection could be seen between the tag and the LSm ring (Fig. $3 \mathrm{G})$. This suggests that the $\mathrm{C}$ terminus of LSm4p forms an elongated domain that protrudes from the LSm ring and is not always visible in individual images. This domain is clearly located at a distance from the Prp24p domain, but not as far away as LSm5p and LSm7p. When additional image processing of U6 snRNPs containing nontagged LSm proteins is performed (Fig. 2B), a small domain protruding from the LSm ring becomes visible. As it is located at a position relative to the Prp24p domain that is similar to that observed with tagged LSm4p, it probably represents the C terminus of LSm4p.

In experiments with tagged LSm2p and LSm3p, the two proteins proposed to be most distant from LSm7p in the LSm ring, the yECitrine tag could not be identified unambiguously in the EM images (data not shown). A possible reason for this is that these two proteins are very close to $\operatorname{Prp} 24 \mathrm{p}$, so that $\operatorname{Prp} 24 \mathrm{p}$ and the tag overlap.

Thus, our data showing that the distance from the Prp24p domain to the yECitrine tag varies according to the identity of the LSm protein, but is relatively constant for a given LSm protein, indicates that the LSm ring is located at a consistent position relative to Prp24p. tags were found closer to the Prp24p domain (Fig. 3E,F). This was most apparent with LSm8p-tagged U6 snRNPs; although the distance between the tag and the Prp24p domain varied somewhat, it was consistently less than that observed with U6 snRNPs containing tagged LSm7p. As LSm8p's C terminus is 30 amino acids downstream of its Sm domain and the yECitrine tag is at the $\mathrm{C}$ terminus, the tag could be located at some distance from the part of LSm8p that is present in the ring.

In the case of LSm4p, the other proposed nearest neighbors of LSm7p, the tag and the Sm domain, are separated by an even larger portion of protein $(\sim 11 \mathrm{kDa})$.

\section{Cross-linking of LSm2p to U6 RNA in the stem linking the LSm and Prp24p binding regions}

In earlier work from this laboratory, a protein was crosslinked to nucleotide G30 at the base of the telestem in the U6 snRNP, but could not be identified (Karaduman et al. 2006). Although this site is within the Prp24p binding region (Fig. 1A), the cross-linked protein was not immunoprecipitated by anti-Prp24p antibodies. Therefore, the protein in question was inferred to be an LSm protein. To determine which of the LSm proteins is in contact with G30, U6 snRNPs with individual LSm proteins tagged with 
yECitrine were UV-irradiated and then disrupted with $1.5 \%$ SDS before immunoprecipitation with anti-yECitrine antibodies. The Northern blot analysis of the coprecipitated U6 snRNAs show that U6 snRNA was efficiently immunoprecipitated only with yECitrine tagged LSm2, LSm3, LSm5, and LSm6 proteins (data not shown), indicating that these proteins are in close contact with the U6 snRNA. The coprecipitated U6 snRNAs were then analyzed by primer extension to map the position of the cross-link. As primer-extension analysis requires annealing of a primer to a sequence downstream from the putative cross-linking site, cross-linking in the region of the established U-rich LSm-binding site at the extreme $3^{\prime}$ end of the U6 snRNA cannot be detected by this technique.

Figure 4 shows the primer-extension analysis of U6 snRNA immunoprecipitated with anti-yECitrine antibodies before (Fig. 4, odd-numbered lanes) and after (Fig. 4, evennumbered lanes) UV irradiation of U6 snRNPs having the yECitrine tag at different LSm proteins. After immunoprecipitation, specific reverse transcriptase stops were detected at nucleotide G30 and, additionally, at nucleotides U28 and
U29 (Fig. 4, lane 2) solely with tagged LSm2p; parallel runs with the yECitrine tag on other LSm proteins (Fig. 4, lanes $4,6,8,10,12,14$ ) and controls without UV irradiation (Fig. 4, lane 1) or without yECitrine-specific antibody (Fig. 4, nonimmune serum [NIS], lane 16) gave negative results. The remaining stops found after UV irradiation correspond to internal RNA-RNA cross-links that are also found in naked U6 RNA.

LSm $2 p$ is thus the only LSm protein that we found UVcross-linked to U6 snRNA outside the pyrimidine-rich, main LSm-binding site at the $3^{\prime}$ end of the RNA, while LSm3p, LSm5p, and LSm6p probably cross-link to the 3' end. The LSm $2 p$ cross-linking takes place at nucleotides 28-30. As U28 and U29 are also cross-linked by UV irradiation to Prp24p (Karaduman et al. 2006), the LSm2p protein would appear to be located at the interface between the LSm ring and Prp24 protein. This correlates well with our EM results obtained with LSm-tagged U6 snRNPs. However, it does not correlate well with previous yeast twohybrid data, which show the strongest Prp24p interactions not with LSm2p, but rather with LSm5p, LSm7p, and LSm8p (Fromont-Racine et al. 2000; Rader and Guthrie 2002), which are located in the ring at sites more remote from the Prp24p domain.

\section{Chemical cross-linking: Fixation of $\mathrm{U} 6$ snRNP in a more compact structure}

To investigate whether U6 snRNPs in solution potentially accommodate more interactions between $\operatorname{Prp} 24 \mathrm{p}$ and the LSm ring, we treated U6 snRNPs after fractionation on a glycerol gradient with gluteraldehyde in order to chemically cross-link them prior to their adsorption onto carbon films. Negativestaining electron microscopy was done as described above. In the overview shown in Figure 5A, it is readily apparent that these particles differ in morphology from the unfixed ones. The U6 snRNP now appears $12 \mathrm{~nm}$ long and $9 \mathrm{~nm}$ wide, and thus considerably more compact. The two substructures (i.e., the round LSm ring and the Prp24p domain), which were clearly identifiable in the unfixed particles, are no longer distinguishable (Figs. 1C,D, 2A,B). Fixation with $p$ formaldehyde yielded very similar images (data not shown).

To determine the structure of the chemically fixed U6 snRNPs in more detail, we further analyzed the images by 
A
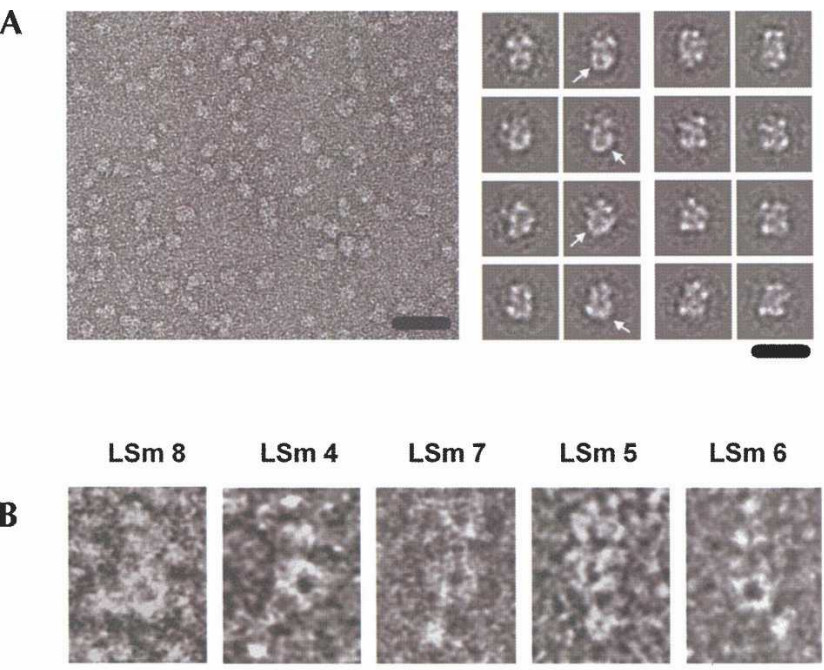

$\operatorname{LSm} 6$
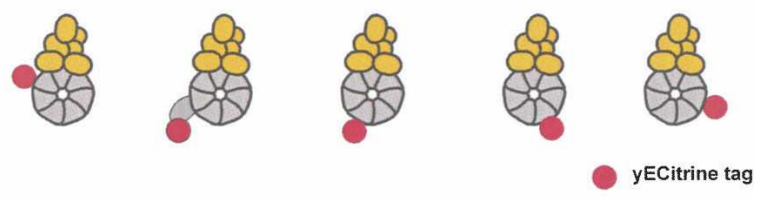

C

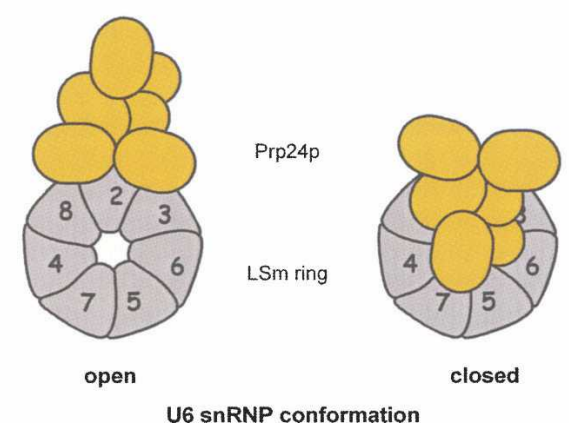

FIGURE 5. Chemical cross-linking yields a more compact form of the U6 snRNP. (A) Electron microscopy of purified U6 snRNP stabilized by chemical cross-linking in solution with glutaraldehyde. Except for the fixation step with glutaraldehyde, all preparation steps were identical to those used to purify the particles shown in Figure 1. (Left) A typical field of glutaraldehyde-stabilized U6 snRNPs. Columns 1-4, class averages of gluteraldehyde-stabilized U6 snRNP particles. Each row shows particles in a similar orientation. In columns 1 and 2, the round features of the LSm ring can be seen (arrows); the images in columns 3 and 4 are more angular, indicative of the Prp24p domain. Scale bars, $20 \mathrm{~nm}$. (B) Summary of EM analysis of yECitrine-labeled U6 snRNPs. Characteristic images of unfixed U6 snRNP particles with yECitrine tags on LSm8p, LSm4p, LSm7p, LSm5p, and LSm6p are shown. Some images are mirrored (see caption to Fig. 3) to illustrate the agreement with the proposed LSm circular sequence in the ring. Below the images, schematic diagrams are shown representing the U6 snRNP particle with yECitrine tags (red disks). (C) Schematic representation of the interaction between Prp24p and the LSm ring for the open (left) and closed (right) form of U6 snRNP. The identities of the LSm proteins in the LSm ring are shown (2-8). The Prp24p domain is represented as arbitrarily arranged protein modules (slightly elongated modules represent the four RRMs and the $\mathrm{N}$ - and C-terminal sequence stretches). Proteins are drawn approximately to scale on the basis of the known sizes of the LSm proteins and the Prp24p domains involved. electronic image processing. Even with a relatively small number of images (e.g., 2000), well-defined class averages were obtained (field and galleries in Fig. 5A). This is a clear indication that the fixed particles are relatively homogeneous with respect to their three-dimensional (3D) structure. In some of the class averages there are hints of the LSm ring and the Prp24 protein domain. That is, they possess a round, smooth outline in the lower part of the image (see columns 1 and 2 of individual images in Fig. $5 \mathrm{~A})$, suggestive of the LSm ring. Further, the upper portion, which clearly partially overlaps with the LSm ring, appears more angular and seems to consist of several small elements, thereby resembling the Prp24p domain (see Figs. 1D, 2A,B). The LSm ring and Prp24 protein seem to overlap to a large extent in the compact form of U6, consistently with Prp24p being in contact with more than one LSm protein in this U6 snRNP conformation.

\section{DISCUSSION}

Our EM experiments show the U6 snRNP in two structural states: (1) a compact, closed state that was seen after chemical fixation in solution, and (2) an open state that predominates when the particles are bound to the carbon carrier film without prior chemical stabilization. On the carbon film, the open form predominates, possibly because in this state the particles bind with greater affinity to the carbon surface, owing to their larger surface area. Weak internal interactions may be broken when the particles are applied to the EM support. Fixing the particles traps the U6 snRNP in the closed form, so that in those preparations the closed form predominates. Recently, it was shown that mild fixation with glutaraldehyde allows the preservation of the solution structure even after binding to the carbon film (Kastner et al. 2008). In that study, a gradient centrifugation fixation protocol, called GraFix, was used. Using the same protocol for fixation of U6 snRNP we also predominantly observed the compact U6 snRNP structure (data not shown). This indicates that the compact, closed U6 snRNP form very likely represents the main form in physiological buffers.

The open form allows better recognition of the Prp24p and the LSm ring, and thus clearer interpretation of EM images of both the wild-type and the LSm-tagged particles (Figs. 1C,D, 3). In most of the open-form images of negatively stained U6 snRNPs, a ring structure with a size characteristic of the Sm ring can be recognized. The Prp24p domain, in contrast, appears angular, with slightly elongated substructures with sizes in the range of that of an RRM domain. This morphology becomes especially clear when the images are processed electronically by averaging the identically oriented domains (Fig. 2; see Results).

To determine the position of each LSm protein relative to Prp24p, and thereby also to answer the question of whether the LSm ring binds the 3' end of the U6 snRNA in 
a unique manner, we developed a new method for the EM localization of proteins in yeast protein/RNP complexes. This method involves the genetic replacement of the wild type by a protein extended at its $\mathrm{C}$ terminus by a yECitrine domain, yielding an additional domain about $4 \mathrm{~nm}$ in size that can be visualized in the electron microscope. Our data demonstrate that the yECitrine domain is well-suited for the labeling of relatively small complexes such as the U6 snRNP. This method should also prove advantageous for studies of other complexes in this size range. An important advantage of this method is that the endogenous protein is completely replaced by the tagged protein, ensuring a very high labeling efficiency. In contrast, labeling of specific structural features with antibodies usually yields a mixture of labeled and unlabeled subpopulations. Larger domains could theoretically be used for labeling as well, and thus this method appears to have the potential to be useful for studying a wide range of macromolecular complexes. However, the smaller the label is, the less likely it will interfere with the function of the labeled protein.

Our labeling experiments clearly show that Prp24 protein is located at a defined position on the LSm ring. This suggests, in turn, that the U6 snRNA also has a consistent position on the ring. According to the currently accepted view, the U6 snRNA is bound through its $3^{\prime}$-terminal sequence to nucleotide-binding pockets on the inner surface of the LSm ring, while the large internal U6 snRNA loop, and many of the nucleotides $5^{\prime}$ of it, are in contact with Prp24p. The telestem of the U6 snRNA (Fig. 1A) lies between these binding sites and may therefore be expected to be close to the interface between the two domains; according to our tagging experiments, LSm2p, LSm3p, and possibly LSm8p are found in this region. Our UV crosslinking experiments also revealed that $\mathrm{LSm} 2 \mathrm{p}$ is located at the RNA-containing interface between the LSm ring and Prp24p, as it can be cross-linked to U6 snRNA nucleotides U28 to G30, which partially also cross-link to Prp24p.

The various distances observed in the U6 snRNP assembled in vivo between the Prp24p domain and the LSm proteins 4, 5, 6, 7, and 8, and the occlusion of LSm proteins 2 and 3, agree with the order of the LSm proteins in the ring as proposed on the basis of protein-protein binding studies (Fig. 5C, 2-3-6-5-7-4-8; Pannone et al. 2001; Zaric et al. 2005). As summarized in Figure 5B, this order is consistent with the Prp24p domain being most distant from LSm5p and LSm7p, less distant from LSm4p and LSm6p, close to LSm8p, and indistinguishable from LSm2p and LSm3p.

In the open form, it appears that only a few LSm proteins (LSm2p and perhaps also LSm3p or LSm8p) are in direct contact with Prp24p at the periphery of the LSm ring. In the closed form, Prp24p appears to be positioned on one of the two "flat" sides of the LSm ring, so that more extensive contact between Prp24p and the LSm proteins can be expected (Fig. 5C). It is possible that the closed form involves contacts between Prp24p and those LSm proteins that interacted with $\operatorname{Prp} 24 \mathrm{p}$ in two-hybrid assays, that is, with LSm5p, LSm7p, and LSm8p. All of these proteins show strong two-hybrid interactions with Prp24p (Fromont-Racine et al. 2000; Rader and Guthrie 2002). Two of them, LSm5p and LSm7p, are widely separated from Prp24p in the open form, but could be proximal in the closed U6 snRNP structure.

Important questions remain concerning the details of how the Prp24 protein and the LSm complex are arranged relative to one another. For example, it is not yet clear which side of the LSm ring binds Prp24p, or how the four RRM modules of Prp24p are oriented. We believe that the protein-tagging method employed here to probe particles with high internal structural homogeneity, such as the U6 snRNP described here, will provide a basis for further three-dimensional structural studies and functional interpretation.

\section{MATERIALS AND METHODS}

\section{Yeast strains, plasmids, and oligonucleotides}

All strains used in this study were derived from Saccharomyces cerevisiae strain YRK3 (Mata trp1- $\Delta 1$, his3- $\Delta$, ura3-52, lys2-801, ade2-101, PRP24::TAP-tag K.l. TRP1 C terminus), which carries a single chromosomal copy of the PRP24 gene containing the TAPtag and the K.l. TRP1 marker at its $\mathrm{C}$ terminus. To introduce a tag at the $\mathrm{C}$ terminus of the LSm proteins, the yECitrine or yECitrine3HA tag was amplified by PCR from plasmid pKT175 or pKT239, respectively (Sheff and Thorn 2004), using the oligonucleotides listed in the Supplementary Materials. The resulting fragments were transformed into YRK3 cells and transformants were selected on solid medium lacking histidine or uracil.

\section{Cell extracts and purification of U6 snRNPs}

Cell extracts were prepared from $8 \mathrm{~L}$ of YPD medium with cells grown to an $\mathrm{OD}_{600}$ of 3.0-5.0. The cells were dropped into liquid nitrogen to make yeast beads with a diameter of 3-6 $\mathrm{mm}$. To obtain U6 snRNPs of sufficient quality for electron microscopy, yeast cells were disrupted by crushing with a mortar grinder (Retsch RM 100) for 12 min. Purification of U6 snRNPs was performed essentially as described earlier (Karaduman et al. 2006). The U6 snRNPs isolated from $8 \mathrm{~L}$ of culture were further subjected to centrifugation in a $10 \%-30 \%$ glycerol gradient at $45,000 \mathrm{rpm}$ for $15 \mathrm{~h}$ at $4^{\circ} \mathrm{C}$ using a Sorvall $\mathrm{TH}-660$ rotor (Karaduman et al. 2006).

\section{UV cross-linking and immunoprecipitation}

For UV cross-linking experiments, affinity-purified U6 snRNPs were irradiated for $2 \mathrm{~min}$ at $254 \mathrm{~nm}$. Protein-protein interactions within the particle were disrupted with $1.5 \%$ SDS (w/v), and specific U6 snRNA-LSm protein cross-links were analyzed by immunoprecipitation with anti-YFP antibodies as described earlier (Karaduman et al. 2006). 


\section{Electron microscopy of U6 snRNP particles}

Negative staining of the particles was carried out by the doublecarbon film method (Kastner et al. 1990). For electron microscopy, snRNP particles were adsorbed on a small piece of carbon film. The film was transferred to a well filled with $2 \%$ uranyl formate in water and incubated for $2 \mathrm{~min}$, and was then lifted from the staining solution with an EM grid covered with a perforated carbon film. A second carbon film floated in another well containing $2 \%$ uranyl formate was placed on top of the first film. After blotting, the grid was stored either at room temperature or in liquid nitrogen. Images were taken at a magnification of 100,000× using a Philips CM20 microscope operated at $120 \mathrm{kV}$. The particles were picked from raw images either manually or semiautomatically by using the software suite Imagic-V (van Heel et al. 1996). Particle images were iteratively classified and aligned by following the "reference-free alignment" protocol (Dube et al. 1993). Class averaging was performed based on multivariate statistical analysis (MSA) of the aligned particle images, followed by hierarchical ascendant classification (HAC) (van Heel 1984). The class averages were based upon about 25 images per class. For stabilization, U6 snRNPs were fixed chemically by incubating for $1 \mathrm{~h}$ on ice with $0.1 \%$ gluteraldehyde, directly after glycerol gradient fractionation and before adsorption onto the carbon film. Image classification and averaging of the U6 projections were carried out as described above, using $\sim 2000$ single-particle images.

\section{SUPPLEMENTAL DATA}

Supplemental material can be found at http://www.rnajournal.org.

\section{ACKNOWLEDGMENTS}

We thank E. Karagöz for preparing LSm7p-tagged U6 particles and M. Killian for technical assistance. We are very grateful to C. Will for critically reading the manuscript. This work was supported by grants from the Federal Ministry of Education and Research, Germany (0311899) and the Sixth Framework Program of the European Union via the Integrated Project "3D Repertoire" to H.S. and by grants from the Fonds der Chemischen Industrie, the Ernst-Jung-Stiftung, and the Deutsche Forschungsgemeinschaft (Lu 294/12-4) to R.L.

Received August 31, 2008; accepted September 15, 2008.

\section{REFERENCES}

Achsel, T., Brahms, H., Kastner, B., Bachi, A., Wilm, M., and Luhrmann, R. 1999. A doughnut-shaped heteromer of human Sm-like proteins binds to the $3^{\prime}$-end of U6 snRNA, thereby facilitating U4/U6 duplex formation in vitro. EMBO J. 18: 5789-5802.

Bae, E., Reiter, N.J., Bingman, C.A., Kwan, S.S., Lee, D., Phillips Jr., G.N., Butcher, S.E., and Brow, D.A. 2007. Structure and interactions of the first three RNA recognition motifs of splicing factor prp24. J. Mol. Biol. 367: 1447-1458.

Bell, M., Schreiner, S., Damianov, A., Reddy, R., and Bindereif, A. 2002. p110, a novel human U6 snRNP protein and U4/U6 snRNP recycling factor. $E M B O \mathrm{~J}$. 21: 2724-2735.

Brow, D.A. 2002. Allosteric cascade of spliceosome activation. Annu. Rev. Genet. 36: 333-360.

Brow, D.A. and Guthrie, C. 1988. Spliceosomal RNA U6 is remarkably conserved from yeast to mammals. Nature 334: 213-218.
Brow, D.A. and Vidaver, R.M. 1995. An element in human U6 RNA destabilizes the U4/U6 spliceosomal RNA complex. RNA 1: 122-131.

Burge, C.B., Tuschl, T., and Sharp, P.A. 1999. Splicing of precursors to mRNAs by the spliceosomes. In The RNA world II (eds. R.F. Gesteland et al.), pp. 525-560. Cold Spring Harbor Laboratory Press, Cold Spring Harbor, NY.

Dube, P., Tavares, P., Lurz, R., and van Heel, M. 1993. The portal protein of bacteriophage SPP1: A DNA pump with 13-fold symmetry. EMBO J. 12: 1303-1309.

Fortner, D.M., Troy, R.G., and Brow, D.A. 1994. A stem/loop in U6 RNA defines a conformational switch required for pre-mRNA splicing. Genes \& Dev. 8: 221-233.

Fromont-Racine, M., Mayes, A.E., Brunet-Simon, A., Rain, J.C., Colley, A., Dix, I., Decourty, L., Joly, N., Ricard, F., Beggs, J.D., et al. 2000. Genome-wide protein interaction screens reveal functional networks involving Sm-like proteins. Yeast 17: 95-110.

Ghetti, A., Company, M., and Abelson, J. 1995. Specificity of Prp24 binding to RNA: A role for Prp24 in the dynamic interaction of U4 and U6 snRNAs. RNA 1: 132-145.

Hermann, H., Fabrizio, P., Raker, V.A., Foulaki, K., Hornig, H., Brahms, H., and Luhrmann, R. 1995. snRNP Sm proteins share two evolutionarily conserved sequence motifs which are involved in Sm protein-protein interactions. EMBO J. 14: 2076-2088.

Jurica, M.S. and Moore, M.J. 2003. Pre-mRNA splicing: Awash in a sea of proteins. Mol. Cell 12: 5-14.

Kambach, C., Walke, S., Young, R., Avis, J.M., de la Fortelle, E., Raker, V.A., Luhrmann, R., Li, J., and Nagai, K. 1999. Crystal structures of two Sm protein complexes and their implications for the assembly of the spliceosomal snRNPs. Cell 96: 375-387.

Karaduman, R., Fabrizio, P., Hartmuth, K., Urlaub, H., and Luhrmann, R. 2006. RNA structure and RNA-protein interactions in purified yeast U6 snRNPs. J. Mol. Biol. 356: 1248-1262.

Kastner, B., Bach, M., and Luhrmann, R. 1990. Electron microscopy of small nuclear ribonucleoprotein (snRNP) particles U2 and U5: Evidence for a common structure-determining principle in the major U snRNP family. Proc. Natl. Acad. Sci. 87: 1710-1714.

Kastner, B., Fischer, N., Golas, M.M., Sander, B., Dube, P., Boehringer, D., Hartmuth, K., Deckert, J., Hauer, F., Wolf, E., et al. 2008. GraFix: Sample preparation for single-particle electron cryomicroscopy. Nat. Methods 5: 53-55.

Kwan, S.S. and Brow, D.A. 2005. The N- and C-terminal RNA recognition motifs of splicing factor Prp24 have distinct functions in U6 RNA binding. RNA 11: 808-820.

Mura, C., Cascio, D., Sawaya, M.R., and Eisenberg, D.S. 2001. The crystal structure of a heptameric archaeal Sm protein: Implications for the eukaryotic snRNP core. Proc. Natl. Acad. Sci. 98: 5532-5537.

Nilsen, T.W. 2003. The spliceosome: The most complex macromolecular machine in the cell? Bioessays 25: 1147-1149.

Pannone, B.K., Do Kim, S., Noe, D.A., and Wolin, S.L. 2001. Multiple functional interactions between components of the Lsm2-Lsm8 complex, U6 snRNA, and the yeast La protein. Genetics 158: 187-196.

Puig, O., Caspary, F., Rigaut, G., Rutz, B., Bouveret, E., BragadoNilsson, E., Wilm, M., and Seraphin, B. 2001. The tandem affinity purification (TAP) method: A general procedure of protein complex purification. Methods 24: 218-229.

Rader, S.D. and Guthrie, C. 2002. A conserved Lsm-interaction motif in Prp24 required for efficient U4/U6 di-snRNP formation. RNA 8: $1378-1392$.

Ryan, D.E., Stevens, S.W., and Abelson, J. 2002. The 5' and 3' domains of yeast U6 snRNA: Lsm proteins facilitate binding of Prp24 protein to the U6 telestem region. RNA 8: 1011-1033.

Séraphin, B. 1995. Sm and Sm-like proteins belong to a large family: Identification of proteins of the U6 as well as the U1, U2, U4, and U5 snRNPs. EMBO J. 14: 2089-2098.

Shannon, K.W. and Guthrie, C. 1991. Suppressors of a U4 snRNA mutation define a novel U6 snRNP protein with RNA-binding motifs. Genes \& Dev. 5: 773-785.

Sheff, M.A. and Thorn, K.S. 2004. Optimized cassettes for fluorescent protein tagging in Saccharomyces cerevisiae. Yeast 21: 661-670. 
Staley, J.P. and Guthrie, C. 1998. Mechanical devices of the spliceosome: Motors, clocks, springs, and things. Cell 92: 315-326.

Stevens, S.W., Barta, I., Ge, H.Y., Moore, R.E., Young, M.K., Lee, T.D., and Abelson, J. 2001. Biochemical and genetic analyses of the U5, U6, and U4/U6 x U5 small nuclear ribonucleoproteins from Saccharomyces cerevisiae. RNA 7: 1543-1553.

van Heel, M. 1984. Multivariate statistical classification of noisy images (randomly oriented biological macromolecules). Ultramicroscopy 13: 165-183.

van Heel, M., Harauz, G., Orlova, E.V., Schmidt, R., and Schatz, M. 1996. A new generation of the IMAGIC image processing system. J. Struct. Biol. 116: 17-24.

Verdone, L., Galardi, S., Page, D., and Beggs, J.D. 2004. Lsm proteins promote regeneration of pre-mRNA splicing activity. Curr. Biol. 14: $1487-1491$.

Vidal, V.P., Verdone, L., Mayes, A.E., and Beggs, J.D. 1999. Characterization of U6 snRNA-protein interactions. RNA 5: 1470-1481.
Vidaver, R.M., Fortner, D.M., Loos-Austin, L.S., and Brow, D.A. 1999. Multiple functions of Saccharomyces cerevisiae splicing protein Prp24 in U6 RNA structural rearrangements. Genetics 153: 12051218.

Will, C.L. and Lührmann, R. 2006. Spliceosome structure and function. In RNA world III (eds. R.F. Gesteland et al.), pp. 369400. Cold Spring Harbor Laboratory Press, Cold Spring Harbor, NY.

Wolff, T. and Bindereif, A. 1993. Conformational changes of U6 RNA during the spliceosome cycle: An intramolecular helix is essential both for initiating the U4-U6 interaction and for the first step of slicing. Genes \& Dev. 7: 1377-1389.

Zaric, B., Chami, M., Remigy, H., Engel, A., Ballmer-Hofer, K., Winkler, F.K., and Kambach, C. 2005. Reconstitution of two recombinant LSm protein complexes reveals aspects of their architecture, assembly, and function. J. Biol. Chem. 280: 1606616075 . 

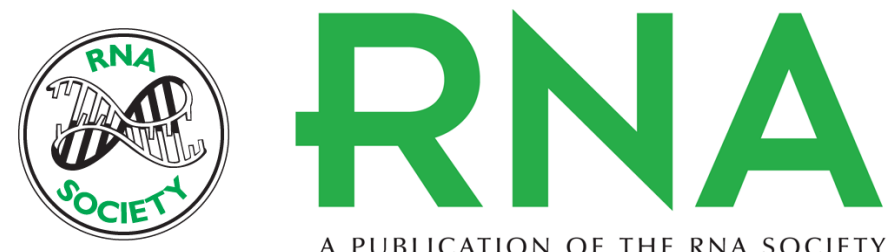

A PUBLICATION OF THE RNA SOCIETY

\section{Structure of yeast U6 snRNPs: Arrangement of Prp24p and the LSm complex as revealed by electron microscopy}

Ramazan Karaduman, Prakash Dube, Holger Stark, et al.

RNA 2008 14: 2528-2537 originally published online October 29, 2008

Access the most recent version at doi:10.1261/rna.1369808

\section{Supplemental http://rnajournal.cshlp.org/content/suppl/2008/10/30/rna.1369808.DC1 \\ Material}

References This article cites 35 articles, 17 of which can be accessed free at: http://rnajournal.cshlp.org/content/14/12/2528.full.html\#ref-list-1

\section{License}

Email Alerting Receive free email alerts when new articles cite this article - sign up in the box at the Service top right corner of the article or click here.

\section{||I||||| Providing Precise Solutions for your research.}

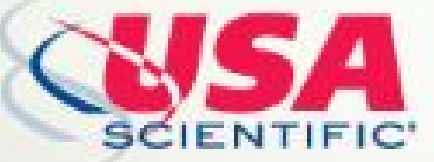

To subscribe to $R N A$ go to:

http://rnajournal.cshlp.org/subscriptions 